\title{
富营养化湖泊蓝藻一芦苇碎屑堆积分解过程及其潜在的共代谢效应
}

\author{
石瑞洁 ${ }^{1,2}$,马 杰 $^{1,2}$, 吕成旭 ${ }^{1,2}$, 许晓光 ${ }^{1,2 * *}$, 党馨逸 ${ }^{1}$, 蒋燕妮 ${ }^{1}$, 叶 子 $^{1}$, 吴懿婷 ${ }^{1}$, 王国祥 ${ }^{1,2}$ \\ ( 1 : 南京师范大学环境学院, 南京 210023) \\ ( 2 : 江苏省地理信息资源开发与利用协同创新中心, 江苏省环境演变与生态建设实验室, 江苏省水土环境生态修复工程 \\ 实验室,南京 210023)
}

\begin{abstract}
摘 要: 为探究富营养化浅水湖泊所富集的有机物对湖泊碳循环和水质的影响, 本研究构建微宇宙系统, 模拟蓝藻和芦 苇碎屑单独分解及混合分解过程. 通过测定各组上覆水营养盐浓度、有机质含量及结构的变化, 揭示富营养化湖泊藻草 残体混合分解过程中养分和有机碳的释放特征. 结果表明, 在实验 $0 \sim 88 \mathrm{~h}$ 内, 在添加相同的碳源条件下, 蓝藻和芦苇混 合处理组总碳 (TC) 释放量显著高于理论值, 表明藻草碎屑混合分解存在共代谢效应. 在培养初期, 沉积物通过共代谢效 应对水质产生了较大的影响, 加速向水体中释放氮、磷物质. 相较单独的植物分解, 混合处理组中总氮 (TN) 、总磷 (TP) 的 最大释放量分别提高了 $13.49 \%$ 和 $26.84 \%$; 通过三维苂光光谱表征的类富里酸苂光强度变化也表明: 较芦苇处理组, 混合 处理组中芦苇的分解速率更快. 在培养开始后, 各处理组均快速转变为厌氧状态, TC、TN、TP 浓度随时间变化总体上呈先 快速上升再逐渐平缓的趋势, 分别在第 $228 、 108$ 和 324 小时达最大值 $(372.4 \pm 2.98) 、(138.45 \pm 2.97)$ 和 $(7.95 \pm 1.11) \mathrm{mg} / \mathrm{L}$. 细菌特异性脂肪酸含量变化表明, 将蓝藻碎屑添加到芦苇碎屑中, 会增加芦苇碎屑中细菌的丰度, 从而提高分解速率, 激 发共代谢效应. 在全球气候变暖的背景下, 随着富营养化湖泊藻类暴发频次增加, 共代谢效应可能还会进一步加强, 对富 营养化湖泊水质将会持续产生影响.
\end{abstract}

关键词: 富营养化湖泊; 共代谢效应; 营养盐; 三维荧光;元素循环

\section{Accumulation and decomposition of cyanobacteria and reed debris in eutrophic lakes and its potential co-metabolism effects *}

Shi Ruijie ${ }^{1,2}$, Ma Jie ${ }^{1,2}$, Lv Chengxu ${ }^{1,2}$, Xu Xiaoguang ${ }^{1,2 * *}$, Dang Xinyi ${ }^{1}$, Jiang Yanni ${ }^{1}$, Ye Zi $^{1}$, Wu Yiting ${ }^{1}$ \& Wang Guoxiang ${ }^{1,2}$

(1: School of Environment, Nanjing Normal University, Nanjing 210023, P.R.China)

(2: Jiangsu Center for Collaborative Innovation in Geographical Information Resource Development and Application, Jiangsu Key Laboratory of Environmental Change and Ecological Construction, Jiangsu Engineering Lab of Water and Soil Eco-remediation, Nanjing 210023, P.R.China)

\begin{abstract}
In order to explore the effect of enriched organic matter in eutrophic shallow lakes on water quality and carbon cycle, in this study, we constructed a microcosm system to simulate the separate decomposition and mixed decomposition of cyanobacteria and reed debris. The changes of nutrient concentration, organic matter content and structure of the overlying water in each treatment were determined to reveal the release characteristics of nutrients and organic carbon during the mixed decomposition process of cyanobacteria and reed debris in eutrophic lakes. The results showed that the total carbon ( TC) release of the mixed treatment was significantly higher than the theoretical value within 0-88 hours of the experiment under the same carbon source addition, indicating that there is a co-metabolism effect during the mixed decomposition of cyanobacteria and reed debris. In the initial stage of the cultivation, sediments accelerated the release of total nitrogen ( TN) and total phosphorus ( TP) to the overlying water and caused a sig-
\end{abstract}

* 2020-09-21 收稿;2020-10-22 收修改稿.

国家水体污染控制与治理科技重大专项(2017ZX07203-003)和国家自然科学基金项目 (42077294, 41703105)联合 资助.

** 通信作者; E-mail:xxg05504118@163.com. 
nificant impact on the water quality via the co-metabolism effect. Compared with the reed treatment, the maximal release of TN and TP in the mixed treatment increased by $13.49 \%$ and $26.84 \%$, respectively. The change of fluorescence intensity of fulvic acid characterized by the three-dimensional fluorescence spectroscopy (EEMs) also showed that the decomposition rate of reed was faster in the mixed treatment group. At the beginning of cultivation, each treatment quickly turned into anaerobic state, and the concentration of TC, TN and TP displayed a trend of dramatical increase and then gradually flattened, with a maximum of $(372.4 \pm 2.98)$, $(138.45 \pm 2.97)$, and $(7.95 \pm 1.11) \mathrm{mg} / \mathrm{L}$ at 228,108 and 324 hours, respectively. The variations of bacterial specific fatty acid showed that the input of Microcystis detritus into Phragmites detritus increased the abundance of decomposing bacteria in Phragmites detritus, and accelerated the decomposition rates, resulting in the co-metabolism of Microcystis and Phragmites carbon. Under the background of global warming, with the increasing frequency of algae outbreaks in eutrophic lakes, the co-metabolism effect may be further strengthened, and the water quality of eutrophic lakes will be affected continuously.

Keywords: Eutrophic lake; co-metabolism effect; nutrient; EEMs; cycle of elements

近年来,湖泊富营养化已成为全球突出的水环境问题之一 ${ }^{[1]}$. 据统计,截止 2019 年,我国已超 $70 \%$ 的湖 泊出现不同程度的富营养化现象 ${ }^{[2]}$. 与一般湖泊相比, 富营养化湖泊中藻华暴发或水草疯长会导致大量藻 草生物碎屑在水一沉积物表面堆积分解, 显著改变湖泊生源要素的循环强度 ${ }^{[3]}$. 以太湖为例, 在风力和湖流 的共同作用下, 大量藻类残体在湖湾、湖滨带、侵蚀漕以及挺水植物群丛等区域与水生植物、外源有机物等 混合堆积腐解,并向水体释放大量营养盐及各类有机物质,在局部区域甚至会引起“湖泛”或“黑水团”等水 环境问题 ${ }^{[4-6]}$. 因此,需要特别关注富营养化湖泊藻草堆积区有机碎屑的分解过程.

随着浅水湖泊富营养化问题的日益加剧, 大量研究关注水生植物残体或藻类碎屑分解对水质的影 响 ${ }^{[-9]}$. 已有结果表明, 不同水生植物在各个季节腐烂分解对水质的影响差异较大, 且春季矿化速率要明显 高于冬季 ${ }^{[10]}$. 挺水植物荠草和芦苇残体衰亡后会快速释放大量营养盐, 同时上覆水中溶解性有机物 (DOM) 的化学结构特征也会随时间的推移而发生变化 ${ }^{[11]}$. 在藻型富营养化湖泊中, DOM 主要来源于藻类 ${ }^{[12]}$, 且藻 源性颗粒有机物降解会快速释放氮磷营养盐和溶解性有机碳 (DOC) ${ }^{[4]}$. 湖泊生物残体分解释放养分和有机 质的过程受多种因素制约 ${ }^{[13]}$, 如植物种类和化学组成、 $\mathrm{pH}$ 大小、微生物群落结构、水文情势等 ${ }^{[10]}$. 初始成分 中氮、磷含量越高的植物残体往往分解速率越快, 而初始纤维素、半纤维素、 $\mathrm{C} / \mathrm{N}$ 和 $\mathrm{C} / \mathrm{P}$ 越高的植物分解速 率越慢 ${ }^{[8]}$. 一般而言, 藻类碎屑的分解速率比水生植物更快, 这与藻类碎屑的理化性质密切相关, 主要是由 于藻类具有更大的比表面积和更高的 $\mathrm{N} / \mathrm{P}^{[14-15]}$. 然而, 这些研究大多针对单一水生植物或藻类分解对水质 的影响,对水生态系统多来源生物碎屑的混合分解过程尚缺乏深人了解.

有研究报道水环境中可能存在类似陆生生态系统的 “共代谢效应”, 即向系统中输人易分解物质 (包括 粪便、调落物以及根系分泌物等) 能激活系统微生物活性, 加速整体有机质代谢速率 ${ }^{[16-17]}$. 在以往的调查中, 课题组发现芦苇和蓝藻混合分解会导致上覆水 $\mathrm{pH}$ 和溶解氧 ( DO) 迅速降低, 且对水质的影响呈现一定阶段 性 ${ }^{[18]}$. Kuzyakov 等研究表明在混合体系中, 如存在多种有机碎屑, 微生物可能会优先分解可利用率较高的 物质, 同时促进微生物大量繁殖 ${ }^{[19]}$. 当易分解物质不再满足微生物需求时, 这些微生物不得不利用相对难 分解的物质, 从而加快整体的代谢速率 ${ }^{[20]}$. 在富营养化湖泊中, 藻类碎屑结构简单, 更容易被微生物利用, 而芦苇碎屑组成相对复杂, 藻一草碎屑在水一沉积物表面大量混合堆积能否通过共代谢效应向水体释放更 多的污染物质尚不清楚.

本研究构建微宇宙系统, 模拟蓝藻和芦苇碎屑单独分解及混合分解过程, 分析比较各处理组上覆水营 养盐浓度、有机质含量及结构的变化规律, 阐明富营养化湖泊藻草残体混合分解过程中碳、氮、磷的释放特 征, 探讨藻草混合分解过程是否存在共代谢效应, 旨在为深人研究富营养化湖泊近岸带不同有机物混合分 解对水环境质量以及物质循环过程的影响提供科学依据.

\section{1 材料与方法}

\section{1 实验样品采集}

芦苇、蓝藻和沉积物均采自太湖西岸沙塘港附近 $\left(31^{\circ} 25^{\prime} 25.38^{\prime \prime} \mathrm{N}, 120^{\circ} 1^{\prime} 54.32^{\prime \prime} \mathrm{E}\right)$. 新鲜蓝藻用清水冲 洗过滤后冷冻干燥, 自封袋密封低温保存; 芦苇洗净杀青后研磨备用; 沉积物过 $150 \mu \mathrm{m}$ 篮混匀, 避光备用, 
沉积物在常温下预培养 3 个月, 使不稳定的有机物质尽量矿化, 同时也使沉积物中营养盐释放, 尽量减少沉 积物对后续培养实验的影响.

\section{2 实验设计}

本研究采用透明 PET 聚酯塑料瓶 ( D = 10 cm, H=20 cm), 向其均匀填充 $100 \mathrm{~g}$ 沉积物 (溶解性总碳 $(\mathrm{TOC})$ 、总氮 $(\mathrm{TN})$ 、总磷 $(\mathrm{TP})$ 含量依次为 $15.1 、 0.91 、 0.72 \mathrm{mg} / \mathrm{g}$ ), 以玻璃棒引流缓慢加人湖水 $500 \mathrm{~mL}$ (湖水 经 $76 \mu \mathrm{m}$ 篮网过笁处理), 静置 1 周, 保持上覆水和沉积物中碳、氮、磷含量处于动态平衡. 实验共设置 4 个 处理组 (内含 1 个对照组), 且每个处理组设置 3 个重复, 为了保持各处理组初始有机碳总量一致, 添加的蓝 藻和芦苇初始干重分别根据其有机碳含量进了换算; 同时, 为了尽量降低沉积物中有机碳作为背景浓度的 影响, 添加了相对较高碳含量的蓝藻和芦苇. (A) 对照组: $100 \mathrm{~g}$ 沉积物; (B) 蓝藻组: $100 \mathrm{~g}$ 沉积物 $+6 \mathrm{~g}$ 蓝藻 (干重) ; (C) 芦苇组: $100 \mathrm{~g}$ 沉积物 $+5.8 \mathrm{~g}$ 芦苇 (干重) ; (D) 混合组: $100 \mathrm{~g}$ 沉积物 $+3 \mathrm{~g}$ 蓝藻 (干重) $+2.9 \mathrm{~g}$ 芦苇 (干重) (初始芦苇中 TOC、TN、TP 浓度分别为 $(394.07 \pm 5.23) 、(15.01 \pm 1.11) 、(2.70 \pm 0.01) \mathrm{mg} / \mathrm{g}$, 初始 蓝藻中 TOC、TN、TP 浓度分别为 $(380.93 \pm 3.45) 、(62.92 \pm 1.55) 、(8.18 \pm 0.86) \mathrm{mg} / \mathrm{g}$. 采用水浴加热, 温度控制 在 $(30 \pm 2)^{\circ} \mathrm{C}$, 于避光条件下连续培养 14 天. 采样时间分别为第 $12 、 24 、 36 、 48 、 60 、 72 、 84 、 96 、 108 、 120 、 132 、$ $156 、 180 、 204 、 228 、 324$ 小时, 每次取出 12 个瓶子 (共计 192 个), 破坏性采样 16 次. 用注射器取出全部上覆 水, 并按规范要求保存 (《水质采样样品的保存和管理技术规定》 $\mathrm{HJ} 493-2009$ ), $24 \mathrm{~h}$ 内完成测试. 沉积物样 品经冷冻干燥后用于测定脂肪酸含量.

\section{3 测定指标与方法}

采样时, 缓慢从各组中取出聚酯塑料瓶, 尽量不扰动上覆水和表层沉积物, 采用便携式水质分析仪 (YSI, 美国) 在水深 $1 / 2$ 处测定水体溶解氧 (DO) 浓度和 $\mathrm{pH}$ 值. 用注射器抽取上覆水 $100 \mathrm{~mL}$, 并经 $0.45 \mu \mathrm{m}$ 玻璃纤维膜抽滤. TN 浓度用碱性过硫酸钾氧化一紫外分光光度法测定 ( GB 11894-1989), TP 浓度采用钼酸 铵分光光度法 (GB 11893-1989) 测定, 总碳 (TC) 和 TOC 浓度采用 multi N/C 3100 型总有机碳分析仪测定 (德国 Jena 公司) (水样预先通过 $0.45 \mu \mathrm{m}$ 玻璃纤维膜, 再根据实际情况进行稀释处理). 取不同处理组上覆 水样品置于四面石英比色血中进行三维荧光光谱扫描, 采用 Hitachi F 7000 型苂光光度计 (日本日立公司) 测定, 光谱仪扫描范围为激发波长/发射波长 $(\mathrm{Ex} / \mathrm{Em})=200 \sim 450 \mathrm{~nm} / 250 \sim 600 \mathrm{~nm}$, 激发波长及发射波长的 带通分别为 5 和 $2 \mathrm{~nm}$, 待扫描结束后使用绘图软件将 $\mathrm{Ex} / \mathrm{Em}$ 所对应的苂光强度进行处理, 绘制等高图.

采用一步法提取并酯化所有的脂质生成脂肪酸甲酯 (FAMEs $)^{[21]}$. 使用气相色谱一质谱联用仪 (GC-MS, Thermo Trace 1310, 美国) 对样品进行定量分析, 该气相色谱仪配备一个安捷伦 DB-5MS 石英毛细管柱 $(60 \mathrm{~m} \times 0.32 \mathrm{~mm} \times 0.25 \mu \mathrm{m})$. 升温程序如下: 初始温度为 $80^{\circ} \mathrm{C}$, 保持 $1 \mathrm{~min}$, 以 $25^{\circ} \mathrm{C} / \mathrm{min}$ 升温至 $189^{\circ} \mathrm{C}$, 再以 $5^{\circ} \mathrm{C} / \mathrm{min}$ 升温至 $310^{\circ} \mathrm{C}$, 保持 $30 \mathrm{~min}$, 进样口温度为 $310^{\circ} \mathrm{C}$. 通过比较样品中脂肪酸的峰面积, 计算每克干样 中的脂肪酸含量 $(\mu \mathrm{g} / \mathrm{g})$; 通过比较各脂肪酸的峰面积与样品中所有脂肪酸的总峰面积, 定性地 (以百分比 计) 计算出各脂肪酸的组成. 利用 $\sum 17$ 脂肪酸含量表征细菌丰度 ${ }^{[22]}$.

\section{4 数据计算分析}

采用一阶动力学方程描述各处理组 TC 浓度的动态变化 ${ }^{[23]}$, 假设释放过程分为快速释放和缓慢释放两 个阶段. 其中, 缓慢释放阶段的速率较慢, 为简化计算, 本研究假设缓慢释放阶段的反应速率近似为 0 , 则方 程可以写成以下形式:

$$
C_{t}=\left(C_{1}\right)_{0} \exp (k \cdot t)+\left(C_{2}\right)_{0}
$$

式中, $k$ 为快速分解阶段 TC 的分解速率, $C_{t}$ 为 $t$ 时刻的 TC 浓度, $\left(C_{1}\right)_{0}$ 和 $\left(C_{2}\right)_{0}$ 分别是 2 个阶段初始的 TC 浓度.

定义 $P E$ 为 $t$ 时刻混合组的浓度与理论值之差:

$$
\begin{gathered}
P E=C_{t(\text { 混合组 })}-C_{t(\text { 理论值 })} \\
C_{t(\text { 理论值 })}=1 / 2\left(C_{t(\text { 滥藻 })}+C_{t(\text { 当蒂 })}\right)
\end{gathered}
$$

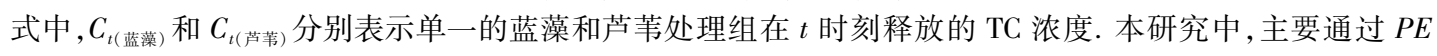
的大小评估两种有机碎屑混合过程是否加快整体溶解性碳的分解速率. 此外, TN、TP 也依照上述处理方法, 并计算 $P E$ 大小, 分别记为 $P E_{\mathrm{TN}}$ 和 $P E_{\mathrm{TP}}$. 
使用 Excel 软件进行数据整理,运用 Origin 2018 软件绘制图形.

\section{2 结果与分析}

\section{1 藻草混合分解过程中上覆水 $\mathrm{DO}$ 浓度及 $\mathrm{pH}$ 变化}

藻草混合分解过程中上覆水 DO 动态变化如图 1a 所示, 各处理组中 DO 浓度均呈先快速降低再逐渐稳 定的趋势. 实验进行到 $60 \mathrm{~h}$ 时, 蓝藻组、芦苇组和混合组 DO 浓度分别下降了 $93.5 \%$ 、86.0\% 和 $88.9 \%$. 各处 理组中上覆水 $\mathrm{pH}$ 在 12 24 h 有所降低, 而后上升直至稳定 (图 $1 \mathrm{~b}$ ). 培养过程中, 蓝藻组、芦苇组和混合组 上覆水 $\mathrm{pH}$ 分别在 6.42 7.9、6.71 7.41 和 6.54 7.91 之间变化. 当培养结束时, 各处理组均呈现弱碱性, 其 中对照组上覆水 $\mathrm{pH}$ 最高,芦苇组 $\mathrm{pH}$ 最低.
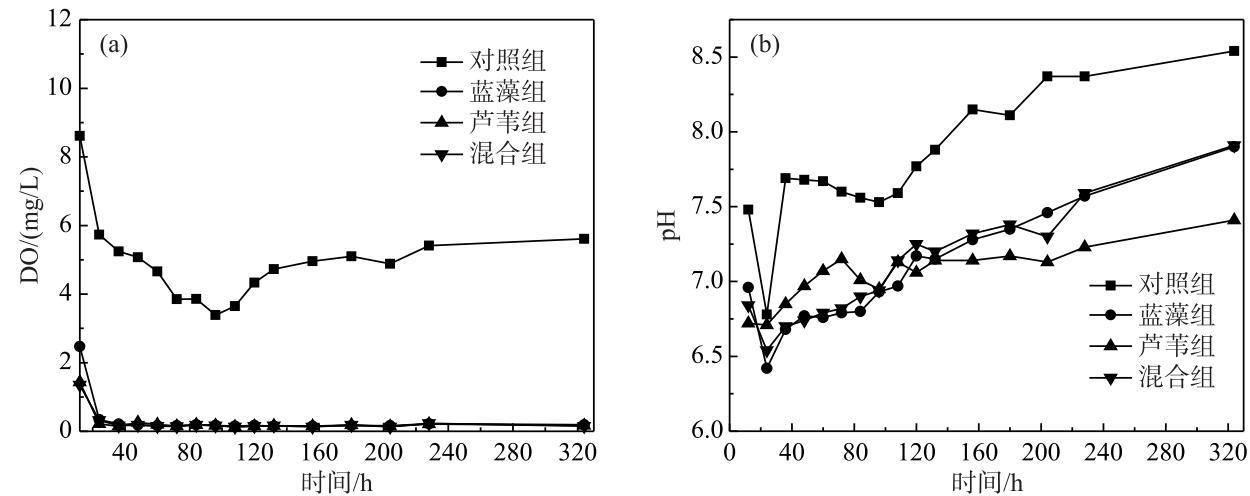

图 1 藻草混合分解过程中上覆水 DO 浓度 $(\mathrm{a})$ 及 $\mathrm{pH}(\mathrm{b})$ 动态变化

Fig. 1 The dynamics of DO concentration(a) and $\mathrm{pH}(\mathrm{b})$ in the overlying water during decomposition processes of cyanobacteria and reeds

\section{2 藻草混合分解过程中上覆水水溶性碳浓度变化}

各处理组上覆水中 TC 浓度变化如图 2 所示. 对照组中 $\mathrm{TC}$ 浓度变化较小, 平均浓度分别为 $(28.56 \pm$ 6.63) $\mathrm{mg} / \mathrm{L}$, 而实验组均呈先上升后趋于平缓的趋势. 蓝藻组 TC 浓度在第 228 小时达最大值 $(372.4 \pm 4.55)$ $\mathrm{mg} / \mathrm{L}$. 芦苇组和混合组的 TC 浓度均在 $36 \mathrm{~h}$ 达最大值, 依次为 $(117.4 \pm 3.99)$ 和 $(221.85 \pm 2.98) \mathrm{mg} / \mathrm{L}$. 结果表 明 $P E_{\mathrm{TC}}$ 值仅在第 $2 \sim 88$ 小时为正 (混合组的释放速率大于理论值). 其中, 在第 25 小时, $P E_{\mathrm{TC}}$ 最大为 27.47 $\mathrm{mg} / \mathrm{L}$, 即 TC 释放量较理论值提高了 $21.69 \%$, 因此, 研究认为草藻混合分解促进了碳的排放.

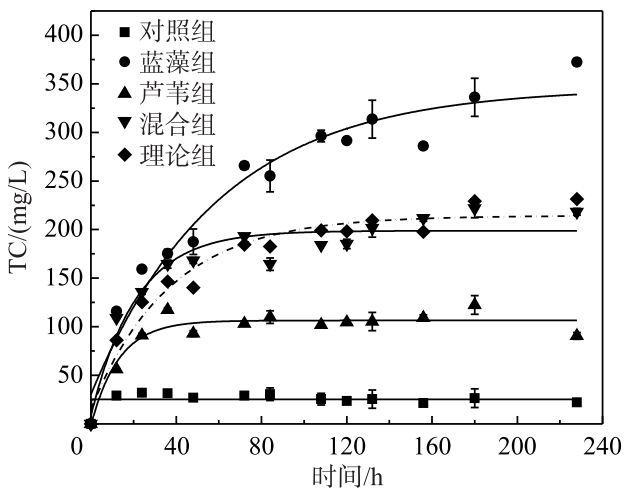

图 2 藻草混合分解过程中上覆水 $\mathrm{TC}$ 浓度动态变化

Fig.2 The dynamics of TC concentration in the overlying water during decomposition processes of cyanobacteria and reeds 
各处理组中上覆水 TIC 和 TOC 浓度变化如图 3 所示. 对照组中 TIC 和 TOC 浓度变化较小, 平均浓度分 别为 $(24.44 \pm 7.06) 、(3.53 \pm 1.50) \mathrm{mg} / \mathrm{L}$, 而实验组均呈先上升后下降的趋势. 蓝藻组 TIC 浓度在第 $228 \mathrm{~h}$ 达 最大值 $(179.00 \pm 4.55) \mathrm{mg} / \mathrm{L}$. 芦苇组和混合组的 TIC 浓度均在第 228 小时达最大值, 依次为 $(85.8 \pm 3.31)$ 和 $(144.4 \pm 2.18) \mathrm{mg} / \mathrm{L}$. 蓝藻组 TOC 浓度在第 156 小时达最大值 $(244.2 \pm 2.38) \mathrm{mg} / \mathrm{L}$, 混合组在第 180 小时达最 大值 $(118.65 \pm 1.87) \mathrm{mg} / \mathrm{L}$.
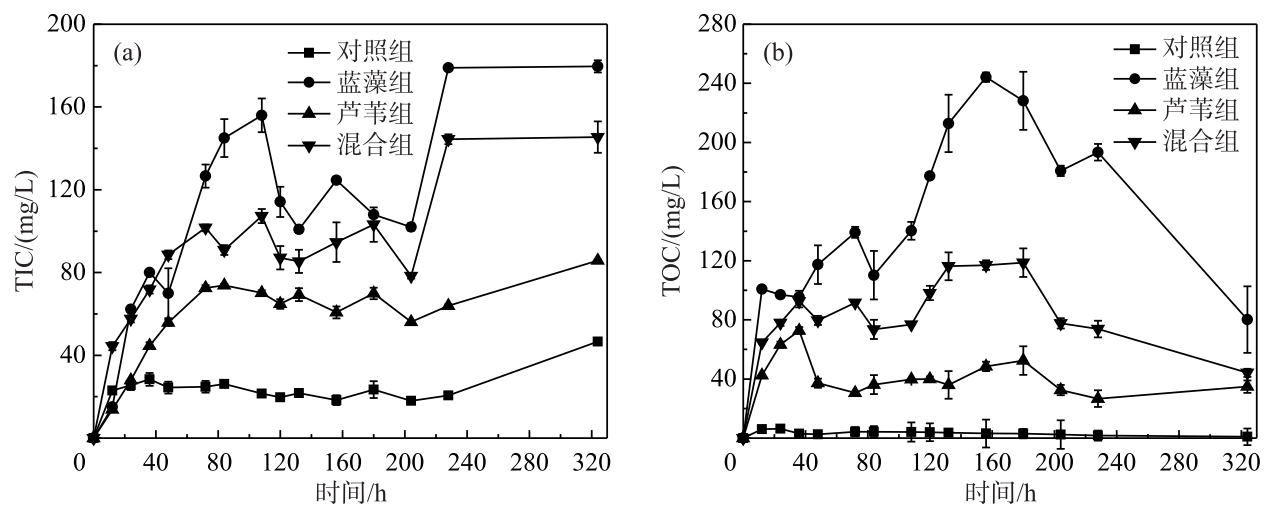

图 3 藻草混合分解过程中上覆水 TIC (a) 和 TOC (b) 浓度动态变化

Fig. 3 The dynamics of TIC (a) and TOC (b) concentrations in the overlying water during decomposition processes of cyanobacteria and reeds

\section{3 藻草混合分解过程中上覆水水溶性氮、磷浓度变化}

藻草混合分解过程中, 各处理组上覆水 TN 浓度变化趋势基本一致,均呈先上升, 后逐渐平稳的趋势 (图 4a). TN 浓度由高到低依次为蓝藻组、混合组和芦苇组. 其中, 蓝藻组和混合组均在第 156 小时达到最大值, 依次为 $(140.3 \pm 3.33)$ 和 $(77.5 \pm 2.97) \mathrm{mg} / \mathrm{L}$, 而芦苇组在第 120 小时达到最大值, 为 $(23.0 \pm 2.23) \mathrm{mg} / \mathrm{L}$. TP 浓 度总体呈先上升后逐渐平稳的趋势 (图 4b). 相较 TN, 实验组 TP 浓度波动较大, 且最大值出现的时间差异 明显, 蓝藻组和混合组分别在第 324 和 228 小时达到最大值, 依次分别为 $(7.95 \pm 2.09)$ 和 $(3.94 \pm 0.10) \mathrm{mg} / \mathrm{L}$, 而芦苇组 TP 浓度在第 180 小时达最大值, 为 $(1.74 \pm 0.97) \mathrm{mg} / \mathrm{L}$.
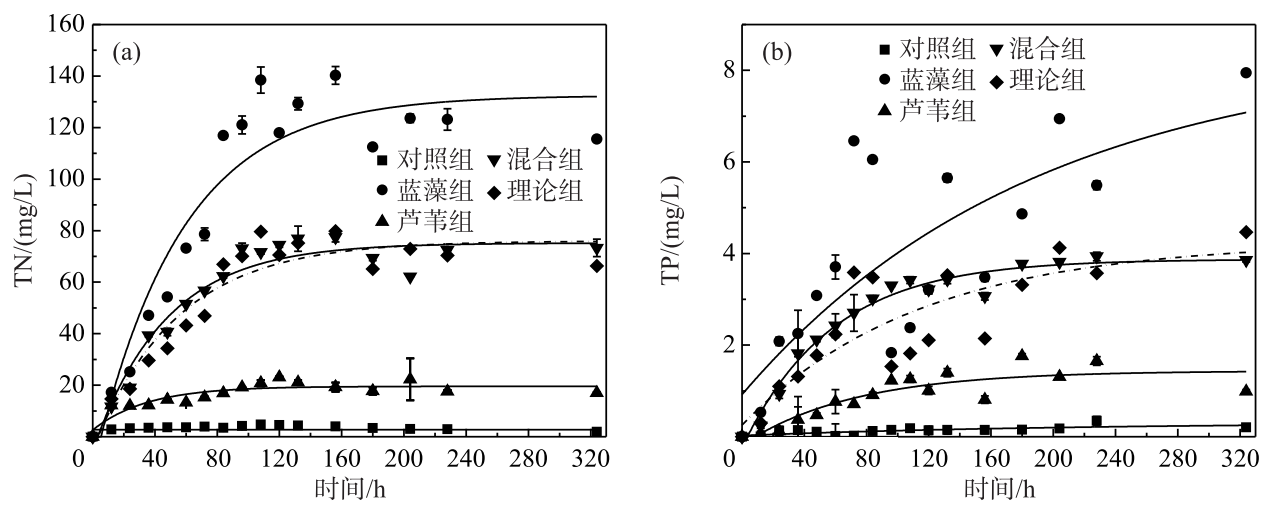

图 4 藻草混合分解过程中上覆水 TN (a) 和 TP $(b)$ 浓度动态变化

Fig.4 The dynamics of TN (a) and TP (b) concentrations in the overlying water during decomposition processes of cyanobacteria and reeds

比较 $P E_{\mathrm{TN}}$ 随时间的变化特征, 注意到 $P E_{\mathrm{TN}}$ 在第 $2 \sim 201$ 小时为正值, 即表明在这期间混合处理组 $\mathrm{TN}$ 的 释放量高于两种碎屑单一处理组的均值. 其中, 在第 28 小时, $P E_{\mathrm{TN}}$ 取得最大值 $3.48 \mathrm{mg} / \mathrm{L}$, 相较理论值提高 
了 $13.49 \%$. 类似地, 计算得到 $P E_{\mathrm{TP}}$ 值在第 $17 \sim 260$ 小时为正值, 其中第 82 小时, $P E_{\mathrm{TP}}$ 值最大, 为 $0.62 \mathrm{mg} / \mathrm{L}$, 相较理论值提高了 $26.84 \%$.

\section{4 藻草混合分解过程中上覆水 DOM 的结构变化}

通过三维荧光光谱分析不同时期(第 108、204、324 小时) 各处理组上覆水 DOM 的结构组成特征(图 5). 结果表明, 分解过程中各处理组三维苂光光谱的苂光峰强度 $(F I)$ 、苂光峰中心位置和苂光指数具有一定差 异性. 各处理组上覆水 DOM 苂光峰类型主要包括类蛋白 A 峰、类蛋白 $\mathrm{C}$ 峰和类富里酸 D 峰. 其中, 蓝藻组 上覆水 DOM 主要以类蛋白质为主, 芦苇组上覆水 DOM 主要以类蛋白质、类富里酸为主, 混合组上覆水 DOM 主要以蛋白质、类富里酸为主. 随着分解过程进行, 所有处理组中类蛋白质的 $F I$ 值均逐渐下降, 类蛋白苂光 峰 $\mathrm{A}$ 和 $\mathrm{C}$ 的 $F I$ 值由大到小依次是: 蓝藻组>混合组>芦苇组. 蓝藻组初期和末期 $F I$ 值分别为 303.8 和 50.4, 芦苇组初期和末期 $F I$ 值分别为 88.6 和 21.4 , 混合组初期和末期 $F I$ 值分别为 166.4 和 25.0 , 而类富里酸的 $F I$ 值有所增加 (表 1). 进一步比较相同时间内芦苇组和混合组中类富里酸 $F I$ 的变化情况, 芦苇组和混合组中 类富里酸 $F I$ 的变化速率分别为 1.27 和 $1.42 \mathrm{~d}^{-1}$.
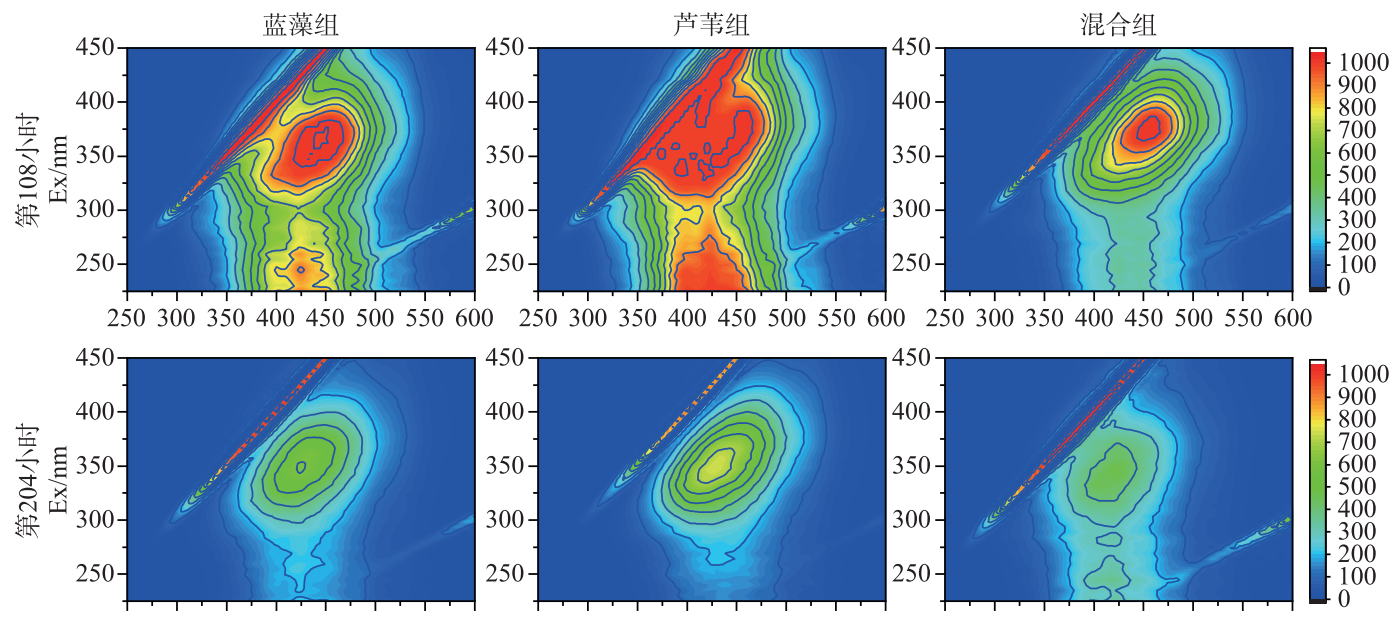

$250300350400450500550600 \quad 250300350400450500550600 \quad 250300350400450500550600$
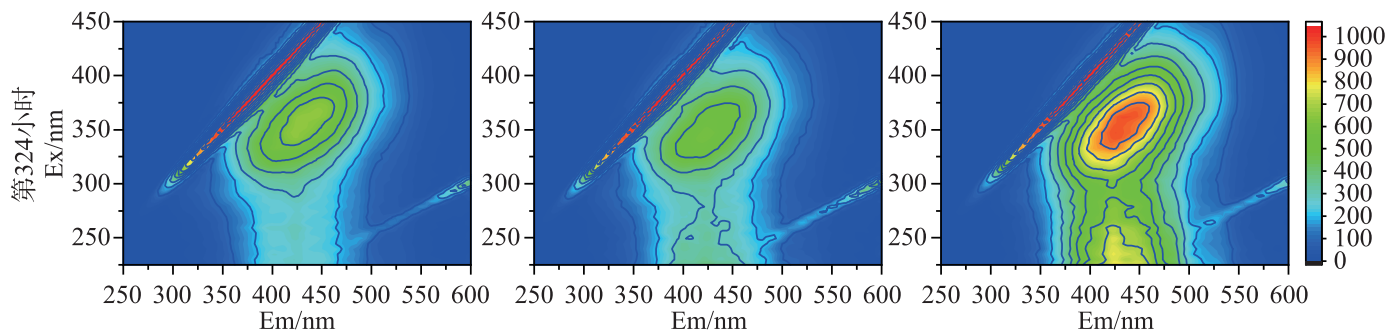

图 5 藻草混合分解不同时期上覆水 DOM 三维菼光光谱

Fig.5 The EEMs of DOM in the overlying water during different decomposition periods of cyanobacteria and reeds

\section{5 细菌特异性脂肪酸含量的变化}

$\sum 17$ 脂肪酸是一种典型的细菌特异性脂肪酸,通常用来阐明细菌对不同有机碎屑的利用情况 ${ }^{[22]}$. 本研 究测定了各处理组在培养前期、中期、后期沉积物中的细菌特异性脂肪酸含量. 结果表明,除芦苇组外,其他 各组中细菌丰度在培养初期后立即增加. 在单一芦苇处理中, 细菌丰度在 $324 \mathrm{~h}$ 后达到峰值 (图 6). 总体而 言,蓝藻组的细菌特异性脂肪酸含量明显高于芦苇组和混合组.

\section{3 讨论}

浅水湖泊中有机物来源十分复杂,包括外源和内源有机碳. 其中, 外源有机碳主要来源于土壤侵蚀和人 
表 1 上覆水中 DOM 苂光特性变化规律

Tab.1 Changes of DOM fluorescence characteristics in the overlying water

\begin{tabular}{|c|c|c|c|c|c|c|c|}
\hline \multirow{2}{*}{ 组别 } & \multirow{2}{*}{ 取样时间 } & \multicolumn{2}{|c|}{ 类蛋白苂光峰 A } & \multicolumn{2}{|c|}{ 类蛋白苂光峰 C } & \multicolumn{2}{|c|}{ 类富里酸苂光峰 D } \\
\hline & & $\mathrm{Ex} / \mathrm{Em} / \mathrm{nm}$ & $F I$ & $\mathrm{Ex} / \mathrm{Em} / \mathrm{nm}$ & $F I$ & $\mathrm{Ex} / \mathrm{Em} / \mathrm{nm}$ & $F I$ \\
\hline \multirow[t]{3}{*}{ 蓝藻组 } & $108 \mathrm{~h}$ & $235 / 350$ & 303.8 & $285 / 350$ & 280.2 & - & - \\
\hline & $204 \mathrm{~h}$ & $240 / 350$ & 286.0 & $290 / 350$ & 216.5 & - & - \\
\hline & $324 \mathrm{~h}$ & $235 / 350$ & 50.4 & $290 / 350$ & 98.6 & - & - \\
\hline \multirow[t]{3}{*}{ 芦苇组 } & $108 \mathrm{~h}$ & $230 / 350$ & 88.6 & $290 / 350$ & 144.4 & $345 / 422.5$ & 471.3 \\
\hline & $204 \mathrm{~h}$ & $240 / 350$ & 42.4 & $290 / 350$ & 106.6 & - & - \\
\hline & $324 \mathrm{~h}$ & $235 / 350$ & 21.4 & $275 / 350$ & 48.2 & $350 / 430.5$ & 746.2 \\
\hline \multirow[t]{3}{*}{ 混合组 } & $108 \mathrm{~h}$ & $225 / 350$ & 166.4 & $290 / 350$ & 146.8 & $360 / 435.5$ & 665 \\
\hline & $204 \mathrm{~h}$ & $240 / 350$ & 91.8 & $290 / 350$ & 157.7 & - & - \\
\hline & $324 \mathrm{~h}$ & $230 / 350$ & 25.0 & $290 / 350$ & 63.4 & $360 / 436$ & 972 \\
\hline
\end{tabular}

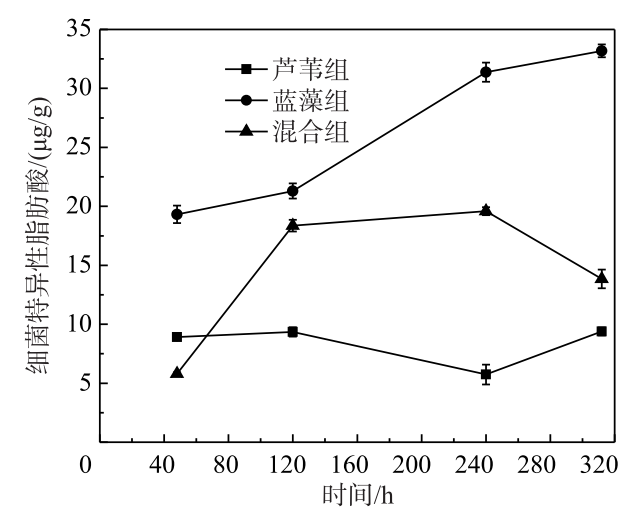

图 6 培养过程中细菌特异性脂肪酸含量的变化

Fig.6 Variations of bacterial specific fatty acid content during incubations
湖河流携带的陆源有机碎屑等; 内源有机碳主要来源于 湖泊水生植物和浮游藻类残体及其分解产物 ${ }^{[24-25]}$. 在以 藻类为主的富营养化湖泊中, 内源是有机碳形成的主要 原因,尤其在水华暴发期间, 大量藻类碎屑在湖滨带、湖 湾以及挺水植物群丛中聚集、沉降和分解, 显著改变湖泊 碳循环强度 ${ }^{[26-27]}$. 本研究通过模拟富营养化湖泊中蓝藻 和芦苇碎屑单独分解及混合分解过程, 揭示了湖泊藻草 残体混合分解过程中碳、氮、磷的释放特征.

本研究中, 在添加相同含量有机碳条件下, 我们计算 得到培养的前 $88 \mathrm{~h}$ 内 $P E_{\mathrm{TC}}$ 为正值, 表明实验初期蓝藻和 芦苇混合分解过程存在共代谢效应,并通过共代谢效应 促进了整体有机碎屑分解,向水体中释放更多溶解性碳. 一般而言, 在有机物戻氧分解过程中, 会同时存在沉积物 中的污染物因浓度梯度向水体中扩散的过程与微生物的 分解作用释放污染物的过程; 本实验中,由于沉积物经过

长期的预培养, 且实验中添加的蓝藻和芦苇的碳含量远远超过了沉积物的背景值, 此外, 实验添加湖水后还 进行了预培养, 保持了碳、氮、磷释放的动态平衡, 因此基本可以排除沉积物释放的影响, 主要为微生物作用 过程. 激发效应的概念最早由土壤学家提出来, 指输人相对 “不稳定碳” 能够改变土壤有机碳矿化过程的一 种机制 ${ }^{[28]}$. 激发效应主要包括微生物分解、动物利用等作用, 而其中的关键微生物过程我们称之为 “共代谢 效应”. 与环境工程中共代谢作用不同的是, 它不是一种在初级能源物质存在时才能进行的某一种或者某一 类平时不能够利用的有机化合物的生物降解过程, 而是讨论一种容易分解的有机物质对另一种难降解物质 的促进作用. 已有研究表明施用粪肥、植物残体调落及释放根系分泌物等过程均会刺激土壤有机质激发强 烈的共代谢效应 ${ }^{[29-30]}$. 尽管共代谢效应已在陆生生态系统中广泛报道, 但直到 2010 年共代谢效应的概念才 被引人水环境中, 因此相关研究仍比较匮乏 ${ }^{[31-32]}$. 相较于芦苇碎屑, 蓝藻颗粒小, 表面积大, 且 N/P 更符合微 生物的生长需求, 因而蓝藻碎屑的周转速率更快 ${ }^{[14-15]}$, 两者物质组成的差异也为共代谢效应的发生提供了 条件. 通常有两种机制来解释共代谢效应的发生过程, 一是 “不稳定碳” 的输人激发了微生物的生长和胞外 酶的活性,进而促进了有机碳的分解过程 ${ }^{[28]}$; 二是 “不稳定碳” 的输人增加了系统中土著微生物的生物量, 提高了整体有机碳的分解速率 ${ }^{[33]}$.

通常情况, 微生物在一些不利于生长的环境中往往处于 “饥饿” 状态, 当加人易分解碳源能快速激发微 生物活性, 促进有机质分解 ${ }^{34]}$. 在培养初期, 混合处理组共代谢效应的增加可能归因于添加蓝藻碎屑激活 
了系统微生物, 进而加速了芦苇碎屑的分解. 有研究指出, 系统中如果存在多种有机质, 微生物可能会优先 分解利用率较高的有机质, 这可能是引起分解进行 $48 \mathrm{~h}$ 后共代谢强度下降的可能原因 ${ }^{[34]}$. 随着蓝藻碎屑被 微生物快速利用, 在 $156 \mathrm{~h}$ 后蓝藻处理组和混合处理组 TC 和 TOC 释放速率逐渐下降. 此外, 水体一部分有 机质在厌氧条件下会产生甲烷和二氧化碳等气体逸出, 这也可能会降低 TC 和 TOC 释放速率 ${ }^{[35]}$. 相较芦苇 组, 混合处理组中 $F I$ 变化速率更大, 说明混合处理组中类富里酸形成速率相对更快, 即表明混合组中芦苇 的分解速率更快. 有研究表明由于有机物分解过程中微生物种类繁多, 并不能简单通过微生物种群变化来 表征共代谢效应的强弱, 而是通过微生物丰度来间接表征有机物的分解快慢, 本研究中, 所有处理组细菌特 异性脂肪酸 ( $\sum 17$ 脂肪酸) 含量在第一周内迅速增加, 说明添加新鲜有机物质可能刺激了细菌生长. 而在前 $156 \mathrm{~h}$, 混合处理中 $\sum 17$ 脂肪酸含量的快速变化过程也证明混合体系中存在共代谢效应. 后续研究还需要进 一步分析酶的活性等来验证该过程. 值得注意的是, 自然生态系统中, 外源碳的输人过程往往存在一定频 率, 例如农田中的粪肥和秸秆等, 森林和草地土壤中的根系分泌有机物和植物调落物等等. 类似的, 在富营 养化湖泊中, 浮游藻类源源不断的堆积分解, 可能会持续不断引起共代谢效应, 促进湖泊生态系统中的碳排 放,因而,进一步阐明富营养化湖泊中由藻类引起的共代谢效应过程对湖泊碳循环具有重要意义.

藻草混合分解除释放碳素外, 还会向上覆水释放大量营养盐, 引起水质恶化等生态问题. 研究对比了混 合组中 TN TP 的释放量与理论值的大小, 混合处理组 TN 在第 2 201 小时的释放量以及 TP 在第 17 260 小时的释放量均明显大于理论值, 说明实验初期蓝藻和芦苇混合分解过程还促进了营养物质的释放, 进一 步加剧了水体的富营养化程度. 以往关于共代谢的研究主要关注体系中碳素变化, 较少关注营养物质的变 化. 所有处理组在实验初期 TN TP 浓度均有所上升, 可能是有机碎屑体内蛋白质、叶绿素等有机氮分解释 放氮素, 与植物体 $\mathrm{C} / \mathrm{N}$ 密切相关 ${ }^{[9,12,36]}$. 实验初期微生物代谢消耗大量氧气, DO 浓度快速下降 (图 1a), 水 体快速处于厌氧环境. 自 $156 \mathrm{~h}$ 后, 各实验组 TN 浓度均逐渐下降, 可能归因于植物在厌氧环境下发生反硝 化过程, 并促使向大气中释放 $\mathrm{N}_{2} 、 \mathrm{~N}_{2} \mathrm{O}^{[3]}$. 至第 228 小时, $\mathrm{TN}$ 浓度基本趋于稳定. 培养结束时, $\mathrm{TN} 、 \mathrm{TP}$ 浓度从 高到低依次为蓝藻组>混合组>芦苇组. 在富营养化湖泊中, 气候变暖一方面会刺激微生物繁殖, 增加有机碳 矿化; 另一方面也会增加湖泊生产力, 引起更多碳沉积. 以太湖为例, 由于气候变暖等因素造成蓝藻物候条 件显著改变, 甚至全年可观测蓝藻水华 ${ }^{[37]}$. 其衰亡沉降后, 延长了与植物碎屑的接触共存时间, 为共代谢提 供了条件 ${ }^{[38]}$. 随着藻类暴发频次进一步增加, 很可能持续激发出正向共代谢效应, 并向水体中释放更多营 养盐,加剧湖泊富营养化以及对全球气候变暖产生正反馈.

本研究证明了在以藻类为主的富营养化湖泊中存在共代谢效应, 能够促使藻草分解向水体中释放更多 的营养盐和有机物. 考虑在全球气候变暖和湖泊富营养化的双重胁迫下, 藻类水华暴发频次和规模均有明 显上升趋势 ${ }^{[39]}$, 共代谢效应对富营养化湖泊的影响可能更大. 本研究利用三维苂光强度评估了相对难分解 物质的形成速率, 进一步从有机物质的化学结构变化来深人分析共代谢效应, 但由于采样间隔时间较长, 并 未捕捉到共代谢强度最大的时刻, 后续研究需要加大采样频率. 基于富营养化湖泊生态系统来源十分复杂, 且易受到干扰, 评估藻类有机碎屑代谢过程引起的共代谢效应的变化特征对于阐明富营养化湖泊碳库的动 态变化以及调控和改善水体水质等问题都具有非常重要的意义.

\section{4 结论}

1) 富营养化湖泊蓝藻芦苇混合分解初期存在明显的共代谢效应, 并且提高了混合碎屑的分解速率. 此 外,草藻混合分解过程也通过共代谢效应加速了水体氮、磷营养盐的释放.

2) 富营养化湖泊中的蓝藻芦苇混合分解过程明显降低了水体 DO 浓度,使得水体长期处于厌氧状态, 极 有可能进一步引起各种水质问题. 在全球气候变暖的背景下,随着富营养化湖泊藻类暴发频次增加,共代谢 效应可能还会进一步加强,对富营养化湖泊水质将会持续产生影响.

\section{5 参考文献}

[ 1 ] Yang LY, Yang XY, Ren LM et al. Mechanism and control strategy of cyanobacterial bloom in Lake Taihu. J Lake Sci, 2019, 31(1) : 18-27. DOI: 10.18307/2019.0102. [杨柳燕, 杨欣妍, 任丽曼等. 太湖蓝藻水华暴发机制与控制对 
策. 湖泊科学, 2019, 31(1): 18-27.]

[ 2 ] Yang TS. Analysis on the development of modern water pollution monitoring technology. Science and Technology \& Innovation, 2018，(13)：154-155. [杨桃生. 浅析现代水污染监测技术的发展. 微计算机信息, 2018，(13) : 154-155.]

[ 3 ] Cao X, Wang GX, Ding XC. The influence of the mixed decomposition of Phragmites australis and algal bloom on water quality. Sichuan Environment, 2019, 38(1) : 6-12. [曹勋, 王国祥, 丁新春. 芦苇和蓝藻混合分解对水质的影响. 四 川环境, 2019, 38(1): 6-12.]

[ 4 ] Krivtsov V, Bellinger EG, Sigee DC. Elemental composition of Microcystis aeruginosa under conditions of lake nutrient depletion. Aquatic Ecology, 2005, 39(2) : 123-134. DOI: 10.1007/s10452-004-6833-5.

[ 5 ] Jiang X, Zhong LX, Wang SH et al. Dissolvable nitrogen variation at water-sediment interface during alga blooming process in Chaohu Lake. China Environmental Science, 2009, 29(11): 1158-1163. [姜霞, 钟立香, 王书航等. 巢湖水华暴发 期水-沉积物界面溶解性氮形态的变化. 中国环境科学, 2009, 29(11) : 1158-1163.]

[6] Huang YX, Zhang XQ, Yang Y et al. Processes of nutrients release from particulate organic matter derived from cyanobacterial blooms in early stage by different microflora. Ecological Science, 2017, 36(5): 55-63. [黄亚新, 张小倩, 杨燕等. 水华初期蓝藻颗粒有机物在不同菌群作用下分解释放营养盐的过程研究. 生态科学, 2017, 36(5): 55-63.]

[ 7 ] Li K, Guan BH, Liu ZW. Experiments on decomposition rate and release forms of nitrogen and phosphorus from the decomposing cyanobacterial detritus. J Lake Sci, 2011, 23(6) : 919-925. DOI: 10.18307/2011.0614. [李柯, 关保华, 刘 正文. 蓝藻碎屑分解速率及氮磷释放形态的实验分析. 湖泊科学, 2011, 23(6): 919-925.]

[ 8 ] Cao PP, Liu MS, Tang JY et al. A comparative study on the decomposition processes among some aquatic plants. Acta Ecologica Sinica, 2014, 34(14) : 3848-3858. DOI: 10.5846/stxb201212011724. [曹培培, 刘茂松, 唐金艳等. 几种水生 植物腐解过程的比较研究. 生态学报, 2014, 34(14): 3848-3858.]

[ 9 ] Zhu MY, Zhu GW, Zhao LL et al. Influence of algal bloom degradation on nutrient release at the sediment-water interface in Lake Taihu, China. Environmental Science and Pollution Research, 2013, 20(3) : 1803-1811. DOI: 10.1007/s11356012-1084-9.

[10] Qi MX, Wang HP, Chen J. Decomposition of Phragmites australis and Typha angustifolia and their effects on the water quality in winter and spring. J Lake Sci, 2017, 29(2) : 420-429. DOI: 10.18307/2017.0218. [戚美侠, 王红萍, 陈杰. 冬、春季芦苇 (Phragmites australis) 和狭叶香蒲 (Typha angustifolia) 的腐解过程及其对水质的影响. 湖泊科学, $2017,29(2): 420-429$.

[11] Xie L, Yang H, Qu XX et al. Dissolved organic matter release of Zizania caduciflora and Phragmites australis from Lake Dianchi. Huanjing Kexue, 2013, 34(9) : 3458-3466. [谢理, 杨浩, 渠晓霞等. 滇池优势挺水植物荠草和芦苇降解过 程中 DOM 释放特征研究. 环境科学, 2013, 34(9) : 3458-3466. ]

[12] Liu FF. Release characteristics and control of organic matter from Chaohu lake algae[Dissertation]. Beijing: University of Chinese Academy of Sciences, 2014. [刘菲菲. 巢湖藻源性有机物释放特征及控制 [ 学位论文]. 北京: 中国科学院 大学, 2014.]

[13] Cao X, Han RM, Zhang TX et al. Decomposition of aquatic plants during winter and its influence on water quality. Journal of Agro-Environment Science, 2015, (2) : 361-369. DOI: 10.11654/jaes.2015.02.021. [曹勋, 韩睿明, 章婷䂀等. 冬 季水生植物分解过程及其对水质的影响研究. 农业环境科学学报, 2015, (2) : 361-369.]

[14] Fu L, Zhao K, Wang GX et al. Temporal and spatial variation of water nutrient level after exogenous nutrient input. Environmental Science, 2014, 35(4) : 1278-1284. DOI: 10.13227/j.hjkx.2014.04.010. [傅玲, 赵凯, 王国祥等. 外源营养 盐输人后水体中营养盐浓度的时空变化. 环境科学, 2014, 35(4) : 1278-1284.]

[15] Zhang J, Wei YC, Wang GX et al. UV-vis spectrum characteristics of phycocyanin in water from Taihu lake. Spectroscopy and Spectral Analysis, 2014, 34(5): 1297. [张静, 韦玉春, 王国祥等. 太湖水体中藻蓝蛋白的紫外-可见吸收光谱 特征分析. 光谱学与光谱分析, 2014, 34(5): 1297.]

[16] Guenet B, Danger M, Harrault L et al. Fast mineralization of land-born C in inland waters: First experimental evidences of aquatic priming effect. Hydrobiologia, 2014, 721(1) : 35-44. DOI: 10.1007/s10750-013-1635-1.

[17] Fontaine S, Mariotti A, Abbadie L. The priming effect of organic matter: A question of microbial competition? Soil Biology and Biochemistry, 2003, 35(6) : 837-843. DOI: 10.1016/S0038-0717(03)00123-8.

[18] Li ZC. The dynamic distribution of organic carbon in water-sediment during the decline of cyanobacteria bloom in Taihu Lake[Dissertation]. Nanjing: Nanjing Normal University, 2019. [李致春. 太湖蓝藻水华衰亡过程中有机碳在水-沉积 物中的动态分布 [学位论文]. 南京: 南京师范大学, 2019.] 
[19] Kuzyakov Y, Bol R. Sources and mechanisms of priming effect induced in two grassland soils amended with slurry and sugar. Soil Biology and Biochemistry, 2006, 38(4) : 747-758. DOI: 10.1016/j.soilbio.2005.06.025.

[20] Wei YY, Cui LJ, Zhang MY et al. Research advances in microbial mechanisms underlying priming effect of soil organic carbon mineralization. Chinese Journal of Ecology, 2019, 38(4) : 1202-1211. DOI: 10.13292/j.1000-4890.201904.008. [魏圆云, 崔丽娟, 张曼胤等. 土壤有机碳矿化激发效应的微生物机制研究进展. 生态学杂志, 2019, 38(4): 1202-1211.]

[21] Abdulkadir S, Tsuchiya M. One-step method for quantitative and qualitative analysis of fatty acids in marine animal samples. Journal of Experimental Marine Biology and Ecology, 2008, 354(1) : 1-8. DOI: 10.1016/j.jembe.2007.08.024.

[22] Xu XG, Li W, Fujibayashi M et al. Predominance of terrestrial organic matter in sediments from a cyanobacteria- blooming hypereutrophic lake. Ecological Indicators, 2015, 50: 35-43. DOI: 10.1016/j.ecolind.2014.10.020.

[23] Dai JH, Sun MY, Culp RA et al. A laboratory study on biochemical degradation and microbial utilization of organic matter comprising a marine diatom, land grass, and salt marsh plant in estuarine ecosystems. Aquatic Ecology, 2009, 43(4) : 825-841. DOI: 10.1007/s10452-008-9211-x.

[24] Wang X, Li ZC, Li X et al. Effect of the decline of cyanobacterial bloom on the organic carbon composition of lake sediment carbon pool. J Lake Sci, 2020, 32 (2) : 346-356. DOI: 10.18307/2020.0212. [汪旋, 李致春, 李翔等. 蓝藻水华 衰亡对沉积物碳库有机碳组分的影响. 湖泊科学, 2020, 32(2) : 346-356.]

[25] Yan XC, Xu XG, Wang MY et al. Climate warming and cyanobacteria blooms: Looks at their relationships from a new perspective. Water Research, 2017, 125: 449-457. DOI: 10.1016/j.watres.2017.09.008.

[26] Liu XS, Shi CF, Xu XG et al. Spatial distributions of $\beta$-cyclocitral and $\beta$-ionone in the sediment and overlying water of the west shore of Taihu Lake. Science of the Total Environment, 2017, 579: 430-438. DOI: 10.1016/j.scitotenv.2016.11.079.

[27] Blagodatskaya E, Kuzyakov Y. Mechanisms of real and apparent priming effects and their dependence on soil microbial biomass and community structure: Critical review. Biology and Fertility of Soils, 2008, 45(2) : 115-131. DOI: 10.1007/ s00374-008-0334-y.

[28] Zhu ZK, Ge TD, Luo Y et al. Microbial stoichiometric flexibility regulates rice straw mineralization and its priming effect in paddy soil. Soil Biology and Biochemistry, 2018, 121: 67-76. DOI : 10.1016/j. soilbio.2018.03.003.

[29] Qiao N, Schaefer D, Blagodatskaya E et al. Labile carbon retention compensates for $\mathrm{CO}_{2}$ released by priming in forest soils. Global Change Biology, 2014, 20(6) : 1943-1954. DOI: 10.1111/gcb.12458.

[30 ] Guenet B, Danger M, Abbadie L et al. Priming effect: Bridging the gap between terrestrial and aquatic ecology. Ecology, 2010, 91(10) : 2850-2861. DOI: 10.1890/09-1968.1.

[31] Bengtsson MM, Attermeyer K, Catalán N. Interactive effects on organic matter processing from soils to the ocean: Are priming effects relevant in aquatic ecosystems? Hydrobiologia, 2018, 822(1) : 1-17. DOI: 10.1007/s10750-018-3672-2.

[32] Fontaine S, Bardoux G, Benest D et al. Mechanisms of the priming effect in a savannah soil amended with cellulose. Soil Science Society of America Journal, 2004, 68(1) : 125-131. DOI: 10.2136/sssaj2004.1250.

[33] Huang WZ, Zhao XL, Zhu JG et al. Priming effect of soil carbon pools. Chinese Journal of Soil Science, 2007, 38(1): 149-154.

[34] Li YH, Li YC, Wang N et al. Formation and control of the harmful ingredients during the decaying process of cyanobacteria from Lake Chaohu. J Lake Sci, 2012, 24(4) : 513-518. DOI: 10.18307/2012.0402. [李永慧, 李玉成, 王宁等. 巢 湖蓝藻死亡衰败过程中典型有害成分形成及控制对策. 湖泊科学, 2012, 24(4) : 513-518.]

[35] Jiao LY, Zhang L, Wang XR et al. Narrow graphene nanoribbons from carbon nanotubes. Nature, 2009, 458(7240): 877-880. DOI: 10.1038/nature07919.

[36] Cao X. Decomposition process of Phragmites australis and cyanobacteria residue and its effect on water quality [ Dissertation]. Nanjing: Nanjing Normal University, 2015. [ 曹勋. 草藻残体分解过程及其对水质的影响 [学位论文]. 南京: 南京师范大学, 2015.]

[37] Shi K, Zhang Y, Xu H et al. Long-term satellite observations of microcystin concentrations in Lake Taihu during cyanobacterial bloom periods. Environmentalence \& Technology, 2015, 10(11) : 6448-6456.

[38 Q Qiao N, Wang J, Xu XL et al. Priming alters soil carbon dynamics during forest succession. Biology and Fertility of Soils, 2019, 55(4) : 339-350.

[39] Shi K, Zhang Y, Zhou Y et al. Long-term MODIS observations of cyanobacterial dynamics in Lake Taihu: Responses to nutrient enrichment and meteorological factors. Scientific Reports, 2017, 7(40326). 